\title{
A Model for Pest Control using Integrated Approach: Impact of Latent and Gestation Delays
}

\author{
M. H. Noor
}

Saralpur High School

Fahad Al Basir ( $\square$ fahadbasir@gmail.com )

Asansol Girls' College https://orcid.org/0000-0003-3744-5524

\section{Research Article}

Keywords: Biopesticides, Predator, Mathematical model, Latent and gestation delay, Hopf bifurcation, Numerical stability analysis.

Posted Date: November 11th, 2021

DOl: https://doi.org/10.21203/rs.3.rs-879070/v1

License: (1) This work is licensed under a Creative Commons Attribution 4.0 International License. Read Full License 


\title{
A model for pest control using integrated approach: impact of latent and gestation delays
}

\author{
M. H. Noor • F. A. Basir
}

the date of receipt and acceptance should be inserted later

\begin{abstract}
In this article, we have established a mathematical model using impulsive differential equations for the dynamics of crop pest management in the presence of a pest with its predator and bio-pesticides. The pest population is divided into two subpopulations, namely, the susceptible pests and the infected pests. In this control process, bio-pesticides (generally virus) infect the susceptible pest through viral infection within the pest and make it infected so that predators can consume it quickly. We assume that pest controlling, using this integrated approach, is a delayed process and thus incorporated latent time of susceptible pest and gestation delay of predator in the model as time delay parameters. The system dynamics have been analyzed using qualitative theory: the existence of the equilibrium points and their stability properties has been derived. Hopf bifurcation of the coexisting equilibrium point is presented for both the delayed and non-delayed system. Detail numerical simulations are performed in support of analytical results and illustrate the different dynamical regimes observed in the system. We have observed that the system becomes free of infection when the latent time of the pest is large. Coexisting equilibrium exists for the lower value of latent delay, and it can change the stability properties from stable to unstable when it crosses its critical value. In contrast, gestation delay affects the stability switches of coexisting equilibrium only. The combined effect of the two delays on the system is shown numerically. Also, viral replication rate, infection rate (from virus to pest) is also significant from the pest management perspective. In summary, both the
\end{abstract}

\footnotetext{
M. H. Noor

Saralpur High School, Saralpur 743135

Murshidabad, West Bengal, India

F. A. Basir*

Department of Mathematics, Asansol Girls' College

West Bengal 713304, India

*Corresponding author, E-mail: fahadbasir@gmail.com.
} 
delay is essential for crop pest management, and pest control will be successful with tolerable delays.

Keywords Biopesticides · Predator · Mathematical model · Latent and gestation delay $\cdot$ Hopf bifurcation · Numerical stability analysis.

\section{Introduction}

Food scarcity gives us the challenge to improve our agricultural tools and techniques at an advanced level and consequently increase the production of crops. Chemicals affect insect populations, pests, and beneficial insects to increase the yield from a crop field. In classical biological control, the pest population is controlled from the region of its origin by the introduction of natural enemies [1]. However, chemical control acts as a hazard to the ecosystem. It makes lots of unwanted harmful effects on other related and nonrelated populations. This kind of problem can be solved if Integrated Pest Management (IPM) is adopted.

IPM is becoming more acceptable among researchers, and its growing applicability in the field by marginal farmers. In an integrated pest control process, we need to reduce the dependency on pesticides by emphasizing the improvement of the applicability of biological control agents. The role of microbial pesticides in the IPM has been recently studied for agriculture, forestry, and public health by various researchers [9]. As an integrated approach, bio-control has a significant role in pest control [14]. On the other hand, there are many animals and birds whose food is the pest population, but these creatures donot hamper the crop. Henceforth they can be used as one of the biological control agents for the control of pest.

In [27], we can find the use of predator populations in removing pest population. Further, we can infect the susceptible pest population by inducing viral or bacterial diseases within the pest. For example, baculovirus usually grows in plants, and this virus has not no direct effect on the production of crops. However, they can be involved to reduce the pest population [29]. Thus, infection to the pest population is one way to eliminate pests from the agriculture field successfully.

Prey-predator type pest control models, with a disease in the prey, (pest) populations are very helpful to describe the dynamics of pest and its natural enemy $[26,28]$. However, there are few theoretical modelling works on the dynamics of pests and their predators and biopesticides [2, 5, 4]. In [2], authors have assumed a mathematical model for integrated pest control using bilinear infection rate. Roy et al. [5] have proposed and analyzed mathematical models for biological pest control, particularly in Jatropha sp. plantation using a virus as a controlling agent. They have used a separate crop population and a logistic type growth for pest population. Later in [4] they have introduced a predator population and shown that integrated pest control is helpful if biopesticide is release in an impulse way. In their paper, Tan and Chen [7] discussed pest control biologically by introducing infected pests. Also, Wang and Song [25] 
used mathematical models to control a pest population by releasing infected pests. All the ecoepidemic models with susceptible prey, infected prey, and the predators can be used to discuss the nature of the susceptible pest, infected pest, and predators but consider time delay as crucial in the biological system dynamics like crop pest control.

Mathematical models using delay differential equations are more realistic as time delays occur in almost all biological systems. In the system under consideration, it is reasonable to consider that the reproduction of the predator after predating the prey is not an immediate process. It has to pass through the gestation of the predator. After predation, some amounts of energy in the biomass of prey assimilate into the predator's energy in the form of biomass. However, this bio-physiological process is not simple. Also, the exchange of prey energy to predator energy is not instantaneous. Different steps are involved in this mechanism. Moreover, there is another time lag present in the pest management system. A time lag exists between the infection of a pest using the virus (biopesticide) and the time it becomes fully infected. This time lag is known as the latent time or latent period of the disease in the pests. In our model, we consider both the gestation and latent delay.

There are few mathematical models for the dynamics of pest control considering time-delay effects. Zhang et al. [8] used a delayed stage-structured epidemic model for pest management. Jana and Kar [10] have considered gestation delay in their prey-predator type model with infection in the prey population. In [11], latent delay was considered in a pest control model using biopesticides, whereas in [13,12], mathematical models were introduced using gestation period as a delay parameter.

In the present study, we develop a mathematical model to study the dynamics of pest controlling system using biopesticides and a predator population. We consider an integrated approach (releasing biopesticides and natural enemy of pest, i.e., predator). Thus the model is an epidemiological model for the dynamics of bio-control of a pest. Then we find out the effects of latent and gestation delays on the system analytically and numerically. To our knowledge, this is the first new work considering both the latent delay and gestation delay in a mathematical model for pest control.

The article is arranged in the following manner. The delay model is derived in section 2 , including some basic mathematical properties. The existence of equilibria and their stability is performed in section 3 and section 4 respectively. Possible occupancy of Hopf bifurcation and its possible controls are discussed in section 5. Detail numerical calculations are presented in section 6 with biological discussion. Finally, we conclude the paper in section 7 with a discussion. 


\section{The mathematical model}

Here, a model is formulated considering four populations, namely the susceptible pest, infected pest, predator, and bio-pesticide. The following assumptions are taken to develop the mathematical model.

A crop plantation system is considered where pest consumes the plant. The plant is the prey, and the pest is the predator. Pest is controlled using bio-pesticides spraying. In this bio-controlling, the pest is made infected as infected pests are less harmful to the plant. Also, predators are incorporated into the system. They uptake the pest population. Therefore, they consume the susceptible pest at a lower rate than consuming the infected pest.

- Let $S(t)$ be the susceptible pest, $I(t)$ be the infected pest, $P(t)$ is the predator (natural enemy of pest) and $V(t)$ is the bio-pesticide (Virus) at any time $t$

- Logistic growth is assumed for plant biomass, with net growth rate $r$ and carrying capacity $K$. Let $\alpha$ and $\beta$ are the predation rates. As predator can catch infected pest easily, we assume $\beta>\alpha$ and $m \leq 1$ is the conversion efficacy.

- There is an infection rate of healthy pest, $\lambda$, due to the use of biopesticide (virus), and $\kappa$ is the virus replication parameter. Finally, $\epsilon$ is the intra-specific competition rate of a predator.

- Let $\delta$ is the mortality rate of infected pest induced infection, and $d$ is the natural mortality rate of a predator.

- Infection of susceptible pest by biopesticide is assumed as a delayed process. Here, $\tau_{1}>0$ is the time delay due to the delayed response of biopesticide. We take another delay $\tau_{2}>0$ due to the gestation of a predator.

Based on the above assumptions, the following mathematical model is derived:

$$
\begin{aligned}
\frac{d S}{d t} & =r S\left(1-\frac{S+I}{K}\right)-\lambda S V-\frac{\alpha S P}{a+S} \\
\frac{d I}{d t} & =\lambda e^{-\delta \tau_{1}} S\left(t-\tau_{1}\right) V\left(t-\tau_{1}\right)-\delta I-\beta I P, \\
\frac{d P}{d t} & =\frac{m \alpha S\left(t-\tau_{2}\right) P\left(t-\tau_{2}\right)}{a+S\left(t-\tau_{2}\right)}+m \beta I\left(t-\tau_{2}\right) P\left(t-\tau_{2}\right)-d P-\epsilon P^{2}, \\
\frac{d V}{d t} & =\kappa \delta I-\mu V
\end{aligned}
$$

Let us assume $\tau=\max \left\{\tau_{1}, \tau_{2}\right\}$ and denote $C$ as the Banach space of continuous functions $\phi:[-\tau, 0] \rightarrow \mathbb{R}_{+}^{4}$ equipped with the sup-norm,

$$
\|\phi\|=\sup _{-\tau \leq \theta \leq 0}\left\{\left|\phi_{1}(\theta)\right|,\left|\phi_{2}(\theta)\right|,\left|\phi_{3}(\theta)\right|,\left|\phi_{4}(\theta)\right|\right\}
$$

where, $\phi=\left(\phi_{1}, \phi_{2}, \phi_{3}, \phi_{4}\right) \in C([-\tau, 0], \mathbb{R})$. For biological reasons, populations always have nonnegative values if they survive, therefore, the initial function 
for model (1) is taken as below:

$$
\begin{aligned}
& S(\theta)=\phi_{1}(\theta), I(\theta)=\phi_{2}(\theta), P(\theta)=\phi_{3}(\theta), \quad V(\theta)=\phi_{4}(\theta) \\
\text { with } & \phi_{i}(\theta) \geq 0, \theta \in[-\tau, 0], \phi_{i}(0)>0, i=1,2,3,4 .
\end{aligned}
$$

\section{Preliminary results}

In this division, some fundamental properties such as positive invariance and boundedness of the solutions of the delayed system (1) are depicted.

Biologically, positivity signifies the survival of the populations. Therefore we require the following theorem.

Theorem 1 All the solution of (1) with the provided initial conditions (2) are positive and are finally bounded.

Proof We can rewrite the system (1) in the following form,

$$
\frac{d X}{d t}=F(X), \quad X=\left(x_{1}, x_{2}, x_{3}, x_{4}\right)^{T}, \quad F=\left(F_{1}, F_{2}, F_{3}, F_{4}\right)^{T},
$$

where $F_{i}$ 's represent the right hand sides of (1), with $x_{1}(t)=S(t), x_{2}(t)=$ $I(t), x_{3}(t)=P(t), x_{4}(t)=V(t)$.

Using the results from [24] and [23], the solution of the system (1) exists in the region $\mathbb{R}_{+}^{4}$ and all solutions remain non-negative for all $t>0$. Indeed, it is easy to check in system (3) that whenever choosing $X(\theta) \in R_{+}$such that $S=0, I=0, P=0, V=0$, it follows

$$
\left.F_{i}(X)\right|_{x_{i}=0, X \in R_{+}^{4}} \geq 0 .
$$

Thus, using the lemma in [24], and the theorem in [23], any solution of (1) with $X(\theta) \in C$, say $X(t)=X(t, X(\theta))$, satisfies $X(\theta) \in R_{+}^{4}$ for all $t \geq 0$. Hence the solution of the system (1) exists in the region $R_{+}^{4}$ and all components remain non-negative for all $t>0$. Therefore, the positive cone $\mathbb{R}_{+}^{4}$ is an invariant region.

To ensure the model remains biologically plausible, both plant and vector populations have to remain bounded during their time evolution. We have the following theorem due to boundedness. To prove the boundedness of $S(t), I(t)$ and $P(t)$, let us define

$$
G(t)=m e^{-\delta \tau_{1}} S(t)+m I\left(t+\tau_{1}\right)+P\left(t+\tau_{2}\right)
$$

Then,

$$
\begin{aligned}
\frac{d G(t)}{d t} & =m e^{-\delta \tau_{1}} \frac{d S}{d t}+m \frac{d I\left(t+\tau_{1}\right)}{d t}+\frac{d P\left(t+\tau_{2}\right)}{d t} \\
& <e^{-\delta \tau_{1}} r S\left[1-\frac{S+I}{K}\right]-\delta I\left(t+\tau_{1}\right)-d P\left(t+\tau_{2}\right) \\
& <e^{-m \tau}\left[(r+m) S-\frac{r S^{2}}{K}\right]-q G(t), \text { where } q=\min \left\{\frac{\delta}{m}, \frac{d}{m}\right\}
\end{aligned}
$$


Now, $(r+m) S-\frac{r S^{2}}{K}$ is a quadratic in $x$ and its maximum value is $\frac{K(r+m)^{2}}{4 r}$. Also the maximum value of $e^{-m \tau}$ is 1 . Using this, we can write

$$
\frac{d G(t)}{d t}<\frac{K(r+m)^{2}}{4 r}-q G(t)
$$

This implies

$$
\limsup _{t \rightarrow \infty} G(t) \leq \frac{K(r+m)^{2}}{4 r q} .
$$

and hence, the $S(t)$ and $I(t)$ are bounded. As $I$ is bounded, using the wellknown comparison principle, from the last equation of $(1), V(t)$ is also bounded.

\subsection{Existence of equilibria}

The model (1) has five equilibria namely (i) the trivial equilibrium $E_{0}(0,0,0,0)$; (ii) the axial equilibrium $E_{1}(K, 0,0,0)$, (iii) biopesticide and infected pest free equilibrium $E_{2}(\bar{S}, 0, \bar{P}, 0)$ where

$$
\bar{P}=\frac{r(K-\bar{S})(a+\bar{S})}{\alpha K}
$$

and $\bar{S}$ is the positive root of

$$
P_{0} S^{3}+P_{1} S^{2}+P_{2} S+P_{3}=0
$$

with coefficients

$P_{0}=-\epsilon r, P_{1}=-2 a r \epsilon+\epsilon r K, P_{2}=-m \alpha^{2} K+d \alpha K-r a^{2} \epsilon+2 \epsilon r K a, P_{3}=\epsilon r K a^{2}+m \alpha K a$.

(iv) the predator-free equilibrium $E_{3}(\tilde{S}, \tilde{I}, 0, \tilde{V})$, where,

$$
\tilde{S}=\frac{\mu}{\lambda e^{-\delta \tau_{1}} \kappa}, \quad \tilde{I}=\frac{\mu\left(K \lambda e^{-\delta \tau_{1}} \kappa-\mu\right) r}{\lambda e^{-\delta \tau_{1}} \kappa(\delta K \lambda \kappa+\mu r)} \quad \tilde{V}=\frac{\delta\left(K \lambda e^{-\delta \tau_{1}} \kappa-\mu\right) r}{\lambda e^{-\delta \tau_{1}}(\delta K \lambda \kappa+\mu r)}
$$

(iv) the coexistence equilibrium, $E^{*}\left(X^{*}, S^{*}, I^{*}, A^{*}\right)$, given by

$$
\begin{gathered}
P^{*}=\frac{\lambda e^{-\delta \tau_{1}} \kappa \delta S^{*}-\mu \delta}{\mu \beta}, \quad V^{*}=\frac{\kappa \delta I^{*}}{\mu}, \\
I^{*}=\frac{\mu \beta d+\epsilon\left(a+S^{*}\right)\left(\lambda e^{-\delta \tau_{1}} \kappa \delta S^{*}-\mu \delta\right)}{\mu m \beta^{2}\left(a+S^{*}\right)},
\end{gathered}
$$

and $S^{*}$ is the positive root of

$$
A_{1} S^{2}+B_{1} S+C_{1}=0 .
$$


$\begin{aligned} A_{1}= & -\delta^{2} \epsilon K \kappa^{2} \lambda^{2} e^{-\delta \tau_{1}}-\delta \epsilon \kappa \lambda e^{-\delta \tau_{1}} \mu r-\beta^{2} m \mu^{2} r, \\ B_{1}= & -a \delta^{2} \epsilon K \kappa^{2} \lambda^{2} e^{-\delta \tau_{1}}+\delta^{2} \epsilon K \kappa \lambda \mu-\alpha \beta \delta K \kappa \lambda e^{-\delta \tau_{1}} m \mu-a \delta \epsilon \kappa \lambda e^{-\delta \tau_{1}} \mu r \\ & +\delta \epsilon \mu^{2} r-a \beta^{2} m \mu^{2} r+\beta^{2} K m \mu^{2} r, \\ C_{1}= & -\beta d \delta K \kappa \lambda \mu+a \delta^{2} \epsilon K \kappa \lambda \mu+\alpha \beta \delta K m \mu^{2}-\beta d \mu^{2} r+a \delta \epsilon \mu^{2} r+a \beta^{2} K m \mu^{2} r .\end{aligned}$

Predator-free equilibrium $E_{3}$ exists when

$$
K \lambda \kappa>\mu e^{\delta \tau_{1}}
$$

Existence condition of infected pest free equilibrium $E_{2}$ is stated below. Here, clearly $P_{0}<0$ and $P_{3}>0$. Thus we have the following proposition.

Proposition 1 Let

$$
\Delta=18 P_{1} P_{2} P_{3} P_{4}-4 P_{2}^{3} P_{4}+P_{2}^{2} P_{3}^{2}-4 P_{1} P_{3}^{3}-27 P_{1}^{2} P_{4}^{2}
$$

be the discriminant of (6). Now, if $\Delta>0$, and if

(a) $P_{1}>0, P_{2}>0$ or $P_{1}<0, P_{2}<0$, then equation (6) has a one positive root,

(b) $P_{2}>0, P_{3}<0, P_{4}<0$ or $P_{2}<0, P_{3}<0, P_{4}>0$, the equation (6) has two positive roots,

(c) $P_{2}>0, P_{3}<0$, then the equation (6) has two positive roots.

Again, noticing $A_{1}<0$ and using the Descartes rule of sign, we derive the following proposition for the existence of coexisting equilibrium $E^{*}$.

Proposition 2 Suppose the discriminant of equation (7) is positive i.e. $B_{1}^{2}-$ $4 A_{1} C_{1}>0$. Now if $B_{1}<0, C_{1}<0$, then (7) has no positive root meaning that there is no feasible endemic equilibrium $E^{*}$. If $B_{1}>0, C_{1}>0$ then there exists a unique endemic equilibrium. But if $B_{1}>0, C_{1}<0$ then there exists exactly two positive roots of (7), consequently two feasible coexisting equilibrium is possible.

\subsection{Characteristic equation}

Characteristic equation of the Jacobian matrix is needed for the stability of equilibria. For this we linearize the system (1) about $E(S, I, P, V)$ as follows:

$$
\frac{d X}{d t}=F X(t)+G X\left(t-\tau_{1}\right)+H X\left(t-\tau_{2}\right)
$$

Here $F, G$ are $4 \times 4$ matrices, given as below:

$$
F=\left[F_{i j}\right]=\left[\begin{array}{cccc}
F_{11} & -\frac{r S}{K} & -\frac{\alpha S}{a+S} & -\lambda S \\
0 & -\delta-\beta P & -\beta I & 0 \\
0 & 0 & -d-2 \epsilon P & 0 \\
0 & \kappa \delta & 0 & -\mu
\end{array}\right] \text {, }
$$




$$
\begin{aligned}
& G=\left[G_{i j}\right]=\left[\begin{array}{cccc}
0 & 0 & 0 & 0 \\
\lambda e^{-\delta \tau_{1}} I & 0 & 0 & \lambda e^{-\delta \tau_{1}} S \\
0 & 0 & 0 & 0 \\
0 & 0 & 0 & 0
\end{array}\right] \text {, } \\
& H=\left[H_{i j}\right]=\left[\begin{array}{cccc}
0 & 0 & 0 & 0 \\
0 & 0 & 0 & 0 \\
\frac{m \alpha P a}{(a+S)^{2}} & m \beta P & H_{33} & 0 \\
0 & 0 & 0 & 0
\end{array}\right]
\end{aligned}
$$

where, $F_{11}=r\left(1-\frac{2 S+I}{K}\right)-\lambda V-\frac{\alpha a P}{(a+S)^{2}}, \quad H_{33}=\frac{m \alpha S}{a+S}+m \beta I$. The characteristic equation of the delay system (1) is given by,

$$
\left|\xi I-F-e^{-\xi \tau_{1}} G-e^{-\xi \tau_{2}} H\right|=0 .
$$

This gives the following characteristic equation,

$$
\begin{aligned}
\phi\left(\rho, \tau_{1}, \tau_{2}\right)= & \rho^{4}+l_{1} \rho^{3}+l_{2} \rho^{2}+l_{3} \rho+l_{4}+e^{-\rho \tau_{1}}\left(b_{1} \rho^{2}+b_{2} \rho+b_{3}\right) \\
& +e^{-\rho \tau_{2}}\left(a_{1} \rho^{3}+a_{2} \rho^{2}+a_{3} \rho+a_{4}\right)+e^{-\rho\left(\tau_{1}+\tau_{2}\right)}\left(q_{1} \rho+q_{2}\right)=0,
\end{aligned}
$$

Coefficients of equation (12) are given in Appendix A.

\section{Stability of equilibria without delay}

In this section, the local stability analysis of the equilibria of the system (1) without delay is studied. For the stability of any equilibrium, we need the distribution of the roots of the characteristic equation (12). From this, we show that the trivial-equilibrium point $E_{0}(0,0,0,0)$ is always unstable.

Next we analyse the stability of virus-free equilibrium $E_{2}(\bar{S}, 0, \bar{P}, 0)$.

4.1 Stability of virus-free equilibrium $E_{2}(\bar{S}, 0, \bar{P}, 0)$

In this case, the Jacobian matrix,

$$
J=\left[J_{i j}\right]_{4 \times 4}=\left[\begin{array}{cccc}
J_{11} & -\frac{r \bar{S}}{K} & -\frac{\alpha \bar{S}}{a+\bar{S}} & -\lambda \bar{S} \\
0 & -\delta-\beta \bar{P} & 0 & \lambda e^{-\delta \tau_{1}} \bar{S} \\
\frac{m \alpha \bar{P} a}{(a+S)^{2}} & m \beta \bar{P} & J_{33} & 0 \\
0 & \kappa \delta & 0 & -\mu
\end{array}\right]
$$


where, $J_{11}=r\left(1-\frac{2 \bar{S}}{K}\right)-\frac{\alpha a \bar{P}}{(a+\bar{S})^{2}}, \quad J_{33}=\frac{m \alpha \bar{S}}{a+\bar{S}}-d-2 \epsilon \bar{P}$.

The characteristic equation at $E_{2}(\bar{S}, 0, \bar{P}, 0)$ is

$$
\xi^{4}+M_{1} \xi^{3}+M_{2} \xi^{2}+M_{3} \xi+M_{4}=0,
$$

with

$$
\begin{aligned}
M_{1}= & -J_{11}-J_{22}-J_{33}-J_{44}, \\
M_{2}= & J_{11} J_{22}-J_{13} J_{31}+J_{11} J_{33}+J_{22} J_{33}+J_{11} J_{44}+J_{22} J_{44}+J_{33} J_{44}-J_{42} J_{24}, \\
M_{3}= & J_{13} J_{22} J_{31}-J_{11} J_{22} J_{33}-J_{11} J_{22} J_{44}+J_{13} J_{31} J_{44}-J_{11} J_{33} J_{44} \\
& -J_{22} J_{33} J_{44}+J_{11} J_{42} J_{24}+J_{33} J_{42} J_{24}, \\
M_{4}= & -J_{13} J_{22} J_{31} J_{44}+J_{11} J_{22} J_{33} J_{44}+J_{13} J_{31} J_{42} J_{24}-J_{11} J_{33} J_{42} J_{24} .
\end{aligned}
$$

For the stability of an equilibrium point we use the Routh-Hurwitz criterion and equation (13) and get the following theorem.

Theorem 2 The virus-free equilibrium $E_{2}$ is asymptotically stable if the following conditions are satisfied

$M_{1}>0, M_{2}>0, M_{3}>0, M_{4}>0, M_{1} M_{2}-M_{3}>0, M_{1} M_{2} M_{3}-M_{3}^{2}-M_{4} M_{1}^{2}>0$.

In the following subsection, we study the stability of predator-free equilibrium $E_{3}$.

4.2 Stability of $E_{3}(\tilde{S}, \tilde{I}, 0, \tilde{V})$ without delay

In this case the Jacobian matric,

$$
L=\left[l_{i j}\right]=\left[\begin{array}{cccc}
l_{11} & -\frac{r \tilde{S}}{K} & -\alpha \tilde{S} & -\lambda \tilde{S} \\
\lambda \tilde{I} & -\delta-d & -\beta \tilde{I} & \lambda \tilde{S} \\
0 & 0 & -d & 0 \\
0 & \kappa \delta & 0 & -\mu
\end{array}\right]
$$

where, $l_{11}=r\left(1-\frac{2 \tilde{S}+\tilde{I}}{K}\right)-\lambda \tilde{V}$. Thus for $\tau_{1}=0=\tau_{2}$, the characteristic equation at $E_{3}$ becomes

$$
(\xi+d) \cdot\left(\xi^{3}+\sigma_{1} \xi^{2}+\sigma_{2} \xi+\sigma_{3}\right)=0
$$

where,

$$
\begin{aligned}
& \sigma_{1}=-\left(l_{11}+l_{22}+l_{33}\right), \\
& \sigma_{2}=l_{11} l_{22}+l_{11} l_{33}+l_{22} l_{33}-l_{12} l_{21}-l_{32} l_{23}, \\
& \sigma_{3}=l_{12} l_{33} l_{21}-l_{11} l_{22} l_{33}-l_{13} l_{32} l_{21}+l_{11} l_{32} l_{23} .
\end{aligned}
$$

Since one of the roots of $(14)$ is $-d<0$, thus using the Routh-Hurwitz criterion on the rest of the roots, we have the following theorem for the stability of $E_{3}$. 
Theorem 3 For $\tau_{1}=0=\tau_{2}$, the predator-free equilibrium $E_{3}(\tilde{S}, \tilde{I}, 0, \tilde{V})$ is stable if and only of

$$
\sigma_{1}>0, \quad \sigma_{2}>0, \quad \sigma_{3}>0, \quad \sigma_{1} \sigma_{2}-\sigma_{3}>0
$$

4.3 Stability of $E^{*}$ without delay

For $\tau_{1}=0=\tau_{2}$, the characteristic equation at endemic equilibrium is

$$
H(\xi)=\xi^{4}+A_{1} \xi^{3}+A_{2} \xi^{2}+A_{3} \xi+A_{4}=0
$$

Employing the Routh-Hurwitz criterion, for the stability of an equilibrium point $E^{*}$, the following conditions need to be satisfied,

$A_{1}>0, A_{2}>0, A_{3}>0, A_{4}>0, A_{1} A_{2}-A_{3}>0, A_{1} A_{2} A_{3}-A_{3}^{2}-A_{4} A_{1}^{2}>0$,

where,

$A_{1}=l_{1}+a_{1}, \quad A_{2}=l_{2}+b_{1}+a_{2}, \quad A_{3}=l_{3}+b_{2}+a_{3}+q_{1}, \quad A_{4}=l_{4}+b_{3}+a_{4}+q_{2}$.

For stability and Hopf bifurcation of $E^{*}$, we have the following theorem.

Theorem 4 The endemic $E^{*}$ with $\tau_{1}=0=\tau_{2}$ is stable if

$$
\begin{aligned}
& A_{2}>0, \quad A_{3}>0, \quad A_{4}>0, \quad A_{1} A_{2}-A_{3}>0, \quad A_{1} A_{2} A_{3}-A_{3}^{2}-A_{4} A_{1}^{2}>0 . \\
& \text { It undergoes a Hopf bifurcation at } \lambda=\lambda^{*} \in(0, \infty) \text { if and only if } \\
& A_{2}\left(\lambda^{*}\right)>0, \quad A_{3}\left(\lambda^{*}\right)>0, \quad A_{4}\left(\lambda^{*}\right)>0, \quad A_{1}\left(\lambda^{*}\right) A_{2}\left(\lambda^{*}\right)-A_{3}\left(\lambda^{*}\right)>0, \\
& \psi\left(\lambda^{*}\right)=0, \text { and } A_{1}^{3} A_{2}^{\prime} A_{3}\left(A_{1}-3 A_{3}\right) \neq\left(A_{2} A_{1}^{2}-2 A_{3}^{2}\right)\left(A_{3}^{\prime} A_{1}^{2}-A_{1}^{\prime} A_{3}^{2}\right) .
\end{aligned}
$$

where $\psi(\lambda)$ is a continuously differentiable function $\psi:(0, \infty) \rightarrow \mathbf{R}$ of $\lambda$ as follows,

$$
\psi(\lambda):=A_{1}(\lambda) A_{2}(\lambda) A_{3}(\lambda)-A_{3}^{2}(\lambda)-A_{4}(\lambda) A_{1}^{2}(\lambda) .
$$

Moreover, at $\lambda=\lambda^{*}$, two eigenvalues $\rho(\lambda)$ of the characteristic equation are purely imaginary, and the other two have purely imaginary parts. Primes denote differentiation concerning the parameter $\lambda$.

Remark 1 Replacing $\lambda$ in the above analysis by any other model parameter (for example, $\alpha, \beta$, etc.), the Hopf bifurcation of endemic equilibrium point can be investigated at the critical value of that parameter. 


\section{Stability analysis with delay}

In this section, we have only provided the local stability of the equilibria $E_{3}$ and $E^{*}$ respectively with delay i.e. when $\tau_{1}>0$ and $\tau_{2}>0$.

For the delay model, the characteristic equation (20) is transcendental in $\xi$ with infinitely many roots. Any steady state, $E(S, I, P, V)$, is locally stable (or unstable) if all the roots of the corresponding characteristic equation have negative real parts (or have positive real parts). Also, Hopf-bifurcating periodic solution persists if at least one purely imaginary root occurs. We shall examine the possible existence of Hopf bifurcation.

\subsection{Stability of $E_{3}$ with delay}

For the stability of $E_{3}$ with delay, three cases will raise: (i) $\tau_{1}>0, \tau_{2}=0$, (ii) $\tau_{1}=0, \tau_{2}>0$ and (iii) $\tau_{1}>0, \tau_{2}>0$.

For $\tau_{1}>0, \tau_{2}=0$, we get the following characteristic equation,

$$
\xi^{3}+\bar{a}_{1} \xi^{2}+\bar{a}_{2} \xi+\bar{a}_{5}+\left[\bar{a}_{3}+\bar{a}_{4} \xi\right] e^{-\xi \tau_{1}}=0 .
$$

where

$$
\begin{aligned}
& \bar{a}_{1}=-\left(l_{11}+l_{22}+l_{33}\right), \quad \bar{a}_{2}=l_{11} l_{22}+l_{11} l_{33}+l_{22} l_{33}, \\
& \bar{a}_{3}=-l_{11} l_{22} l_{33}, \quad b_{1}=-l_{12} m_{21}-l_{32} m_{23}, \\
& b_{2}=l_{12} l_{33} m_{21}-l_{13} l_{32} m_{21}+l_{11} l_{32} m_{23}
\end{aligned}
$$

For Hopf bifurcation to occur, we have to show that there exists a purely imaginary eigenvalue. Suppose $\xi=i w\left(\tau_{1}\right)$ is a purely imaginary root of the equation (20), then we have

$$
-i w^{3}-\bar{a}_{1} w^{2}+i \bar{a}_{2} w+\left(\bar{a}_{4} i w+\bar{a}_{3}\right)\left(\cos w \tau_{1}-i \sin w \tau_{1}\right)+\bar{a}_{5}=0 .
$$

Grouping real and imaginary parts, we get the following equations,

$\bar{a}_{1} w^{2}-\bar{a}_{5}=\bar{a}_{3} \cos w \tau_{1}+\bar{a}_{4} w \sin w \tau_{1}, \quad w^{3}-\bar{a}_{2} w=\bar{a}_{4} w \cos w \tau_{1}-\bar{a}_{3} \sin w \tau_{1}$.

Squaring and adding the real and imaginary parts we get,

$$
w^{6}+\left(\bar{a}_{1}^{2}-2 \bar{a}_{2}\right) w^{4}+\left(\bar{a}_{2}^{2}-2 \bar{a}_{1} \bar{a}_{5}-\bar{a}_{4}^{2}\right) w^{2}+\left(\bar{a}_{5}^{2}-\bar{a}_{3}^{2}\right)=0 .
$$

Putting $\theta=w^{2}$, the equation (23) is reduced to

$$
H(\theta)=\theta^{3}+S_{1} \theta^{2}+S_{2} \theta+S_{3}=0,
$$

where,

$$
S_{1}=\bar{a}_{1}^{2}-2 \bar{a}_{2}, \quad S_{2}=\bar{a}_{2}^{2}-2 \bar{a}_{1} \bar{a}_{5}-\bar{a}_{4}^{2}, \quad S_{3}=\bar{a}_{5}^{2}-\bar{a}_{3}^{2} .
$$

Now if $S_{3}<0$ holds then equation (24) has at least one positive root. Let $\theta_{0}$ be the least positive root of (24) for which the characteristic equation (20) has pair of purely imaginary roots $\pm i \sqrt{\theta_{0}}$. 
From the equation (22), writing $\sqrt{\theta_{0}}=w$, we get the values of $\tau_{1}$ for which (24) has positive roots is

$$
\tau_{1}^{k}=\frac{1}{w} \cos ^{-1}\left[\frac{\bar{a}_{4} w^{4}-\left(\bar{a}_{2} \bar{a}_{4}-\bar{a}_{1} \bar{a}_{3}\right) w^{2}-\bar{a}_{3} \bar{a}_{5}}{\bar{a}_{4}^{2} w^{2}+\bar{a}_{3}^{2}}\right]+\frac{2 k \pi}{w}, \quad k=0,1,2, \ldots,
$$

Let

$$
\tau_{10}^{*}=\min _{k \geq 0}\left\{\tau_{1}^{k}\right\}, w_{0}=w\left(\tau_{10}^{*}\right) .
$$

Then we have the following proposition.

Proposition 3 Suppose that the interior equilibrium point $E^{*}$ exists and is locally asymptotically stable for $\tau_{1}=0$ i.e. (17) is satisfied. Now, if either, $S_{3}<0$, then $E^{*}$ is asymptotically stable when $\tau<\tau_{10}^{*}$ and unstable when $\tau>\tau_{10}^{*}$, where

$$
\tau_{10}^{*}=\frac{1}{w_{0}} \arccos \left[\frac{\bar{a}_{4} w_{0}^{4}-\left(\bar{a}_{2} \bar{a}_{4}-\bar{a}_{1} \bar{a}_{3}\right) w_{0}^{2}-\bar{a}_{3} \bar{a}_{5}}{\bar{a}_{4}^{2} w_{0}^{2}+\bar{a}_{3}^{2}}\right] .
$$

Thus, when $\tau_{1}=\tau_{10}^{*}$, Hopf bifurcation occurs, i.e. a family of periodic solution bifurcates at $E^{*}$ as $\tau$ passes through the critical value $\tau_{10}^{*}$ provided the transversality condition, $3 \omega_{0}^{4}+2 S_{1} \omega_{0}^{2}+S_{2} \neq 0$ is satisfied.

Proof Clearly, for the proof of the theorem we have to verify only the transversality condition.

Denoting $\xi=\xi\left(\tau_{1}\right)$ and differentiating (20), we obtain

$$
\left(\frac{\mathrm{d} \xi\left(\tau_{1}\right)}{\mathrm{d} \tau_{1}}\right)^{-1}=-\frac{3 \xi^{2}+2 \bar{a}_{1} \xi+\bar{a}_{2}}{\xi\left(\xi^{3}+\bar{a}_{1} \xi^{2}+\bar{a}_{2} \xi+\bar{a}_{5}\right)}+\frac{\bar{a}_{4}}{\xi\left(\bar{a}_{4} \xi+\bar{a}_{3}\right)}-\frac{\tau_{1}}{\xi},
$$

which leads to

$$
\begin{aligned}
& \operatorname{sign}\left\{\operatorname{Re}\left(\frac{\mathrm{d} \xi}{\mathrm{d} \tau_{1}}\right)_{\tau_{1}=\tau_{1}^{k}}\right\} \\
& =\operatorname{sign}\left\{\operatorname{Re}\left(\frac{\mathrm{d} \xi}{\mathrm{d} \tau_{1}}\right)_{\tau_{1}=\tau_{1}^{k}}^{-1}\right\} \\
& =\operatorname{sign}\left\{3 \omega_{0}^{4}+\left(2 \bar{a}_{1}^{2}-4 \bar{a}_{2}\right) \omega_{0}^{2}+\bar{a}_{2}^{2}-2 \bar{a}_{1} \bar{a}_{5}-\bar{a}_{4}^{2}\right\} \\
& =\operatorname{sign}\left\{3 \omega_{0}^{4}+2 S_{1} \omega_{0}^{2}+S_{2}\right\} .
\end{aligned}
$$

Thus

$$
\operatorname{Re}\left[\frac{\mathrm{d} \xi}{\mathrm{d} \tau_{1}}\right]_{\tau_{1}=\tau_{10}^{*}} \neq 0 \Leftrightarrow 3 \omega_{0}^{4}+2 S_{1} \omega_{0}^{2}+S_{2} \neq 0 .
$$

Hence the transversality condition is verified, and consequently the occurrence of Hopf bifurcation at $\tau_{1}=\tau_{10}^{*}$ is established.

Remark 2 Stability of $E_{3}$ for $\tau_{1}=0, \tau_{2}>0$ and for $\tau_{1}>0, \tau_{2}>0$ can be studied using the analysis in the following sub-section. 
5.2 Stability of $E^{*}$ with delay

In this section we check the stability switches of endemic equilibrium for with delay i.e. when $\tau_{1}>0$ and $\tau_{2}>0$. We will study three cases, namely (i) $\tau_{1}>0, \tau_{2}=0$, (ii) $\tau_{1}=0, \tau_{2}>0$ and (iii) $\tau_{1}>0, \tau_{2}>0$.

(i) For $\tau_{1}>0, \tau_{2}=0$, the characteristic equation at the endemic equilibrium $E^{*}$ (derived from $\left.(12)\right)$ is

$$
\begin{aligned}
\phi\left(\xi, \tau_{1}, 0\right)= & \xi^{4}+C_{1} \xi^{3}+C_{2} \xi^{2}+C_{3} \xi+C_{4}+ \\
& e^{-\xi \tau_{1}}\left[D_{1} \xi^{2}+D_{2} \xi+D_{3}\right]=0
\end{aligned}
$$

where,

$$
\begin{gathered}
C_{1}=\left(a_{1}+l_{1}\right), \quad C_{2}=\left(a_{2}+l_{2}\right), \quad C_{3}=\left(a_{3}+l_{3}\right), \\
C_{4}=\left(a_{4}+l_{4}\right), \quad D_{1}=b_{1}, \quad D_{2}=b_{2}+q_{1}, \quad D_{3}=b_{3}+q_{2} .
\end{gathered}
$$

The terms $a_{1}, a_{2}, . ., a_{4}, b_{1}, b_{2}$ etc. are given in Appendix-A.

For the stability changes to occur, we have to show that there exists a pair of purely imaginary roots of the characteristic equation for a critical value of $\tau_{1}>0$. Suppose that there exists a purely imaginary root say $i \theta$ of equation (30).

We substitute $\xi=i \theta$ in (30) and then separating real and imaginary parts we finally obtain

$$
\begin{aligned}
& D_{1} \sin \theta \tau_{1}+D_{2} \cos \theta \tau_{1}=-\theta^{4}+C_{2} \theta^{2}-C_{4}, \\
& D_{1} \cos \theta \tau_{1}-D_{2} \sin \theta \tau_{1}=C_{1} \theta^{3}-C_{3} \theta .
\end{aligned}
$$

First we take the square and then add the above two equations to get,

$$
l^{8}+\omega_{1} l^{6}+\omega_{2} l^{4}+\omega_{3} l^{2}+\omega_{4}=0 .
$$

Again we substitute $\theta^{2}=l$ in (33) and get the following equation

$$
l^{4}+\omega_{1} l^{3}+\omega_{2} l^{2}+\omega_{3} l+\omega_{4}=0 .
$$

The coefficients of (34) are

$$
\begin{aligned}
& \omega_{1}=C_{1}^{2}-2 C_{2}, \quad \omega_{2}=C_{2}^{2}+2 C_{4}-2 C_{1} C_{3}, \\
& \omega_{3}=-2 C_{2} C_{4}+C_{3}^{2}-D_{1}^{2}, \quad \omega_{4}=C_{4}^{2}-\left(D_{1}^{2}+D_{2}^{2}\right) .
\end{aligned}
$$

Let us define $H(l)$ by

$$
H(l)=l^{4}+\omega_{1} l^{3}+\omega_{2} l^{2}+\omega_{3} l+\omega_{4} .
$$

Given that $H(0)=\omega_{4}<0$ and also note that $\lim _{l \rightarrow \infty} H(l)=\infty$. Hence, there exists an $l_{0} \in(0, \infty)$ so that $H\left(l_{0}\right)=0$. Thus we have the following lemma.

Lemma 1 The equation (34) has at least one positive root when $\omega_{4}<0$. 
Without loss of generality, we assume that the equation (34) has four positive roots. We denote them as $l_{r}^{*}, r=1,2,3,4$. Then (33) also has four positive roots, $\theta_{r}=\sqrt{z_{r}^{*}}, r=1,2,3,4$. From equation (31), the values of $\tau_{1}$ is calculated as

$$
\begin{gathered}
\tau_{1}^{n}=\frac{1}{\theta_{r}} \cos ^{-1}\left[\frac{\theta_{0}^{2} D_{2}\left[C_{1} \theta_{0}^{2}-C_{3}\right]+\left(D_{1} \theta_{0}^{2}-D_{3}\right)\left[\theta_{0}^{4}-C_{2} \theta_{0}^{2}+C_{4}\right]}{\left(D_{1} \theta_{0}^{2}-D_{3}\right)^{2}+D_{2}^{2} \theta_{0}^{2}}\right]+\frac{2 \pi n}{\theta_{r}}, \\
\quad r=1,2,3,4 \text { and } n=0,1,2,3, \ldots
\end{gathered}
$$

Thus, we establish that $\pm i \theta_{r}$ is a pair of purely imaginary roots of (30). Let us write the following

$$
\tau_{1}^{*}=\tau_{1}^{n}{ }_{r_{0}}^{n}=\min _{n \geq 0,1 \leq r \leq 4}\left\{\tau_{1}^{n}\right\}, \quad \theta_{0}=\theta_{r_{0}}, \quad l_{0}=l_{r_{0}}^{*} .
$$

Then we can have the following results.

Theorem 5 Suppose that the $E^{*}$ is stable without delay. Then for $\omega_{4}<$ 0 , the steady state $E^{*}$ is locally asymptotically stable for $\tau_{1}<\tau_{1}^{*}$, and it is unstable when $\tau_{1}>\tau_{1}^{*}$. Furthermore, the occurrence of Hopf bifurcation at $E^{*}$ is confirmed when $\tau_{1}=\tau_{1}^{*}$ provided that the transversality condition $4 \theta_{0}^{6}+A_{1} \theta_{0}^{4}+A_{2} \theta_{0}^{2}+A_{3} \neq 0$ is satisfied.

Proof First part of the theorem is immediate from the above analysis. Thus, we have to prove the last part of the theorem only.

We Differentiate (30) with respect to $\tau_{1}$ to obtain

$$
\frac{d \tau_{1}}{d \xi}=\frac{4 \xi^{3}+3\left(C_{1} \xi^{2}+2 C_{2} \xi+C_{3}\right)}{D_{2} \xi^{3}+D_{3} \xi^{2}+D_{4} \xi} e^{\xi \tau_{1}}+\frac{2 D_{2} \xi+D_{3}}{D_{2} \xi^{3}+D_{3} \xi^{2}+D_{4} \xi}-\frac{\tau_{1}}{\xi} .
$$

Considering equation (31), it is easy to show the following result,

$$
\begin{aligned}
\operatorname{Sgn}\left[\frac{d(\operatorname{Re} \xi)}{d \tau_{1}}\right]_{\tau_{1}=\tau_{1}^{*}} & =\operatorname{Sgn}\left[\operatorname{Re}\left(\frac{d \xi}{d \tau_{1}}\right)^{-1}\right]_{\xi=i \xi_{0}}, \\
& =\operatorname{Sgn}\left[\frac{4 \xi_{0}^{6}+\omega_{1} \xi_{0}^{4}+\omega_{2} \xi_{0}^{2}+\omega_{3}}{D_{3}^{2} \xi_{0}^{2}+\left[-D_{2} \xi_{0}^{2}+D_{4}\right]^{2}}\right],
\end{aligned}
$$

Since $D_{3}^{2} \xi_{0}^{2}+\left[-D_{2} \xi_{0}^{2}+D_{4}\right]^{2}$ is always positive. Also, from equation (36) we see that $H^{\prime}\left(l_{0}\right) \neq 0$ if $4 \xi_{0}^{6}+\omega_{1} \xi_{0}^{4}+\omega_{2} \xi_{0}^{2}+\omega_{3} \neq 0$. Thus it follows that

$$
\operatorname{Sgn}\left[\frac{d(\operatorname{Re} \xi)}{d \tau_{1}}\right]_{\tau_{1}=\tau_{1}^{*}} \neq 0 .
$$

(ii) The analysis for $\tau_{2}>0, \tau_{1}=0$ is given in Appendix B. (iii) For $\tau_{1}>$ $0, \tau_{2}>0$, we provide the result without proof in the following theorem.

Theorem 6 Suppose that the non-delayed system is asymptotically stable for $\tau_{2} \in\left(0, \tau_{2}^{*}\right)$. Now, if $\omega_{4}<0$ holds then there exists $\tau^{*}$ for which stability switch occurs at $E^{*}$ when $\tau_{1}$ passes the critical value $\tau^{*}$. Furthermore, $E^{*}$ will undergo a Hopf bifurcation when $\tau_{1}=\tau^{*}$, provided that

$$
\left[\frac{d(\operatorname{Re} \xi)}{d \tau_{1}}\right]_{\tau_{1}=\tau^{*}}>0 .
$$


Table 1 Values of parameters used in numerical calculation for system.

\begin{tabular}{|c|l|c|}
\hline Parameter & \multicolumn{1}{|c|}{ Short description } & Values \\
\hline$r$ & growth rate of pest & 0.1 \\
$K$ & maximum density of pest & 1 \\
$\lambda$ & infection rate of susceptible pest & -0.2 \\
$d$ & natural mortality rate of pest & 0.012 \\
$\delta$ & additional mortality of infected pest & 0.02 \\
$\alpha$ & predator catching rate of susceptible pest & 0.01 \\
$\beta$ & predator catching rate of infected pest & 0.09 \\
$n$ & lysis of predator due to competition & 5 \\
$m$ & conversion factor for predator & 0.6 \\
$\mu$ & decay rate of virus & 0.1 \\
$a$ & half-saturation constant & 0.5 \\
\hline
\end{tabular}

In this case the characteristic equation is

$$
\begin{aligned}
\phi\left(\rho, \tau_{1}, \tau_{2}\right)= & \rho^{4}+l_{1} \rho^{3}+l_{2} \rho^{2}+l_{3} \rho+l_{4}+e^{-\rho \tau_{1}}\left(b_{1} \rho^{2}+b_{2} \rho+b_{3}\right) \\
& +e^{-\rho \tau_{2}}\left(a_{1} \rho^{2}+a_{2} \rho+a_{3}\right)+e^{-\rho\left(\tau_{1}+\tau_{2}\right)}\left(q_{1} \rho^{2}+q_{2} \rho+q_{3}\right)=0,
\end{aligned}
$$

Thus comparing the results in [32] (Theorem 5 and Theorem 6 of [32]), a formal proof of the Theorem 6 can be analysed. A similar result can be provided for $\tau_{1}$ when $\tau_{2} \in\left(0, \tau_{2}^{*}\right)$.

\section{Numerical simulations}

This section provides numerical examples to analyze the results and interpretations we have made in the previous sections.

Numerical solution of the model system without delay is plotted in Figure 1. From this figure it is confirmed that for $\lambda=0.012$ the system is asymptotically stable (confirmed by Theorem 4). All the system populations oscillate initially but finally converges to the endemic equilibrium $E^{*}(2.212,0.8425,22.67,6.71)$. For the higher value of the infection rate $\lambda=0.01388$, stable periodic orbit is observed. Hopf bifurcation diagram of coexisting equilibrium $E^{*}$ is plotted in Figure 2. It indicates that system population bifurcates into the periodic solution at $E^{*}$ when $\lambda$ crosses its critical value $\lambda^{*}=0.01388$. It is also obvious that for higher values of $\lambda, E^{*}$ is not stable, but $E_{3}$ exists and is unstable. Also, we have plotted the bifurcation of endemic equilibrium, taking $\beta$ as the main parameter (see Figure 3). In Figure 4, bifurcation diagram of the system is plotted for the value of delay $\tau_{1}$. The system (1) has periodic orbits when $\tau_{1}$ lies in the range $\tau_{1} \in(0,50)$. That is endemic equilibrium $E^{*}$ is stable for higher values of delay $\tau_{1}$. It is clear from Figure 4 that the stability switches of the endemic equilibrium $E^{*}$ in terms of time delay $\tau_{1}$ at two points, nearly at $\tau \approx 3.86$ days and $\tau \approx 41.25$ days from stable to unstable and from unstable to stable, respectively. Bifurcation plot of Figure 5 shows that gestation delay destabilises the system when its value is larger than $\tau_{2}^{*}=4.78$. 

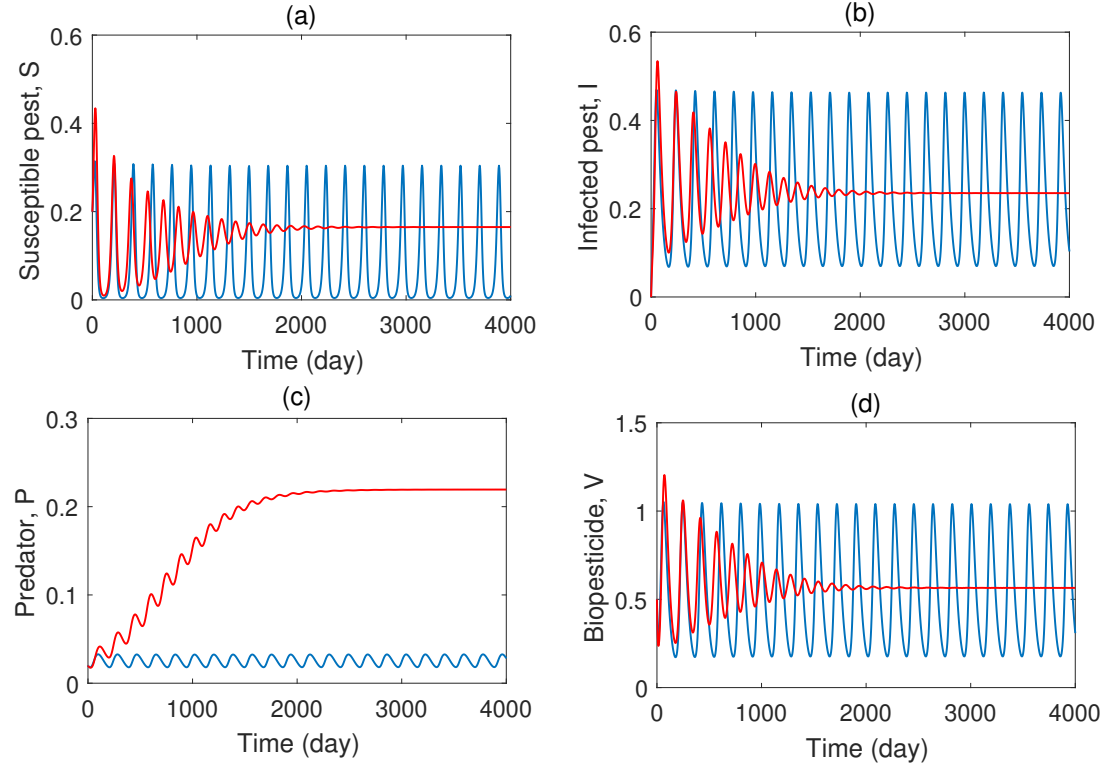

Fig. 1 Numerical solution of the system without delay is plotted using the values of the parameters from Table 1 except $\lambda=0.01$ (red line) and $\lambda=0.1363$ (blue line).

(a)

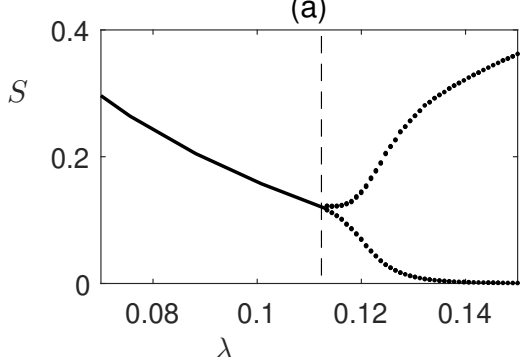

(c)

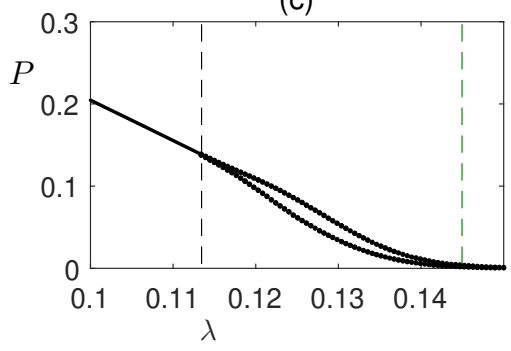

(b)

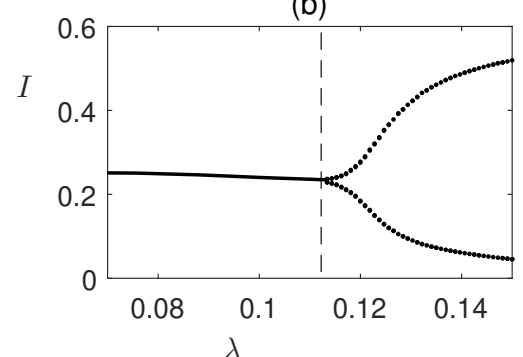

(d)

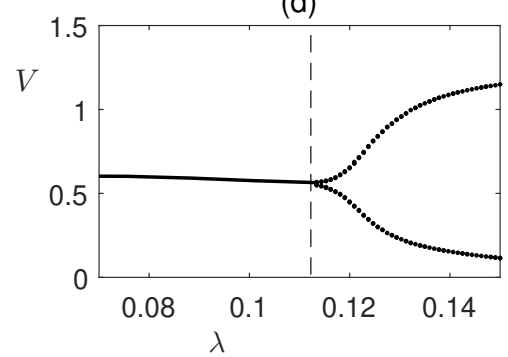

Fig. 2 Hopf bifurcation of non-delay model taking $\lambda$ as bifurcation parameter. 

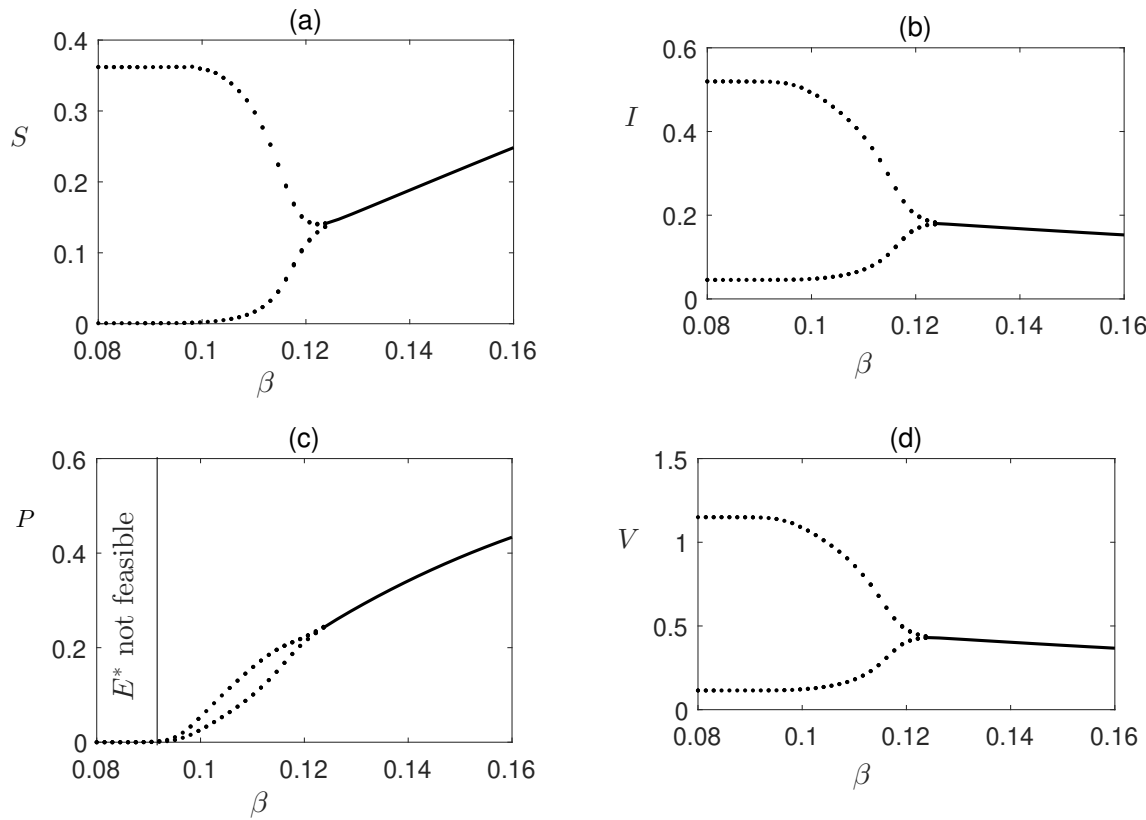

Fig. 3 Hopf bifurcation of non-delay model taking $\beta$ as bifurcation parameter. Values of the parameters same as Figure 1 except $\lambda=0.1363$.

(a)

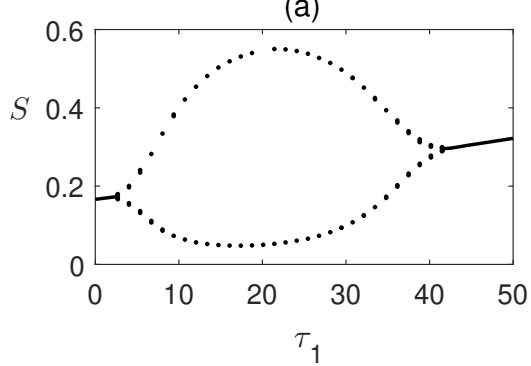

(c)

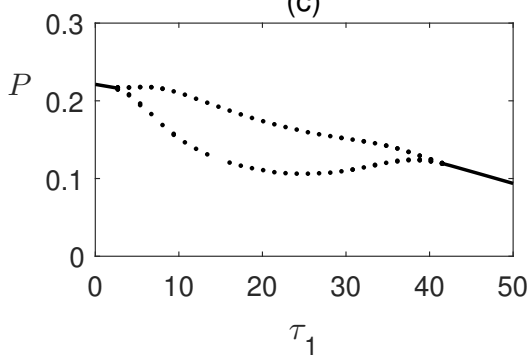

(b)

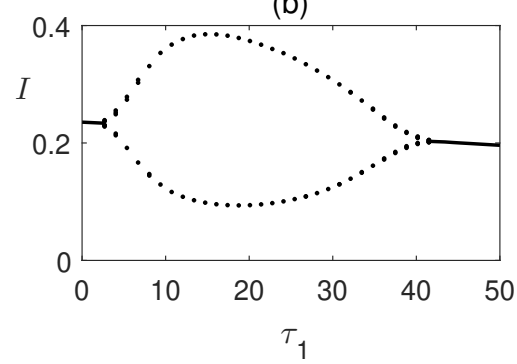

(d)

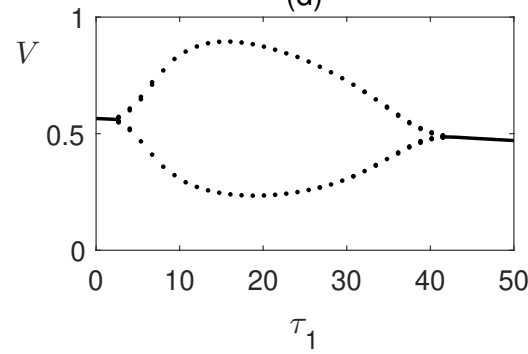

Fig. 4 Hopf bifurcation of endemic equilibrium $E^{*}$ of the delay model taking $\tau_{1}$ as bifurcation parameter and $\tau_{2}=0$. Parameters values are same as Figure 2. 
(a)

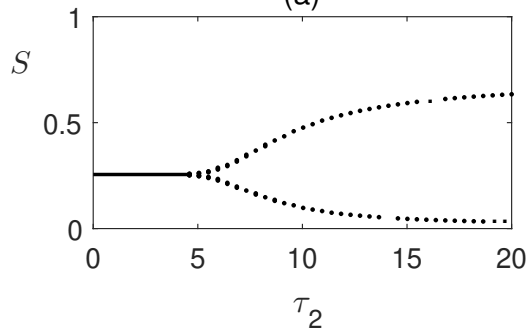

(c)

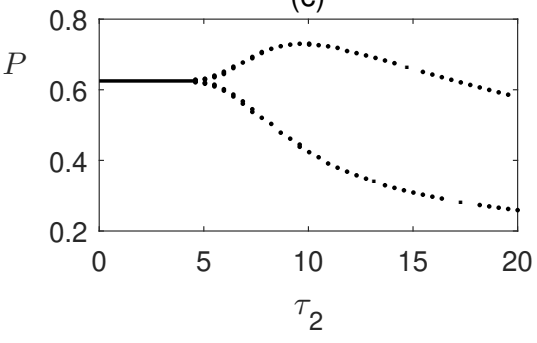

(b)

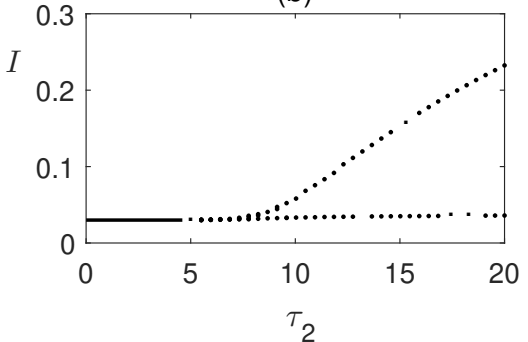

(d)

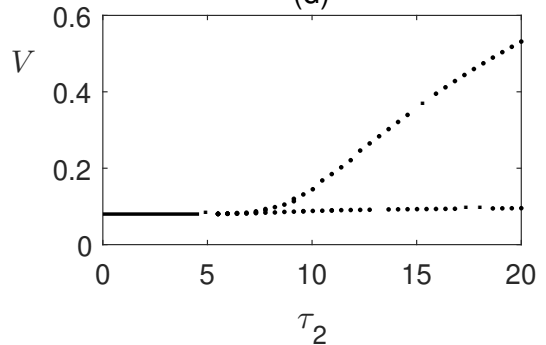

Fig. 5 Hopf bifurcation of the delay model taking $\tau_{2}$ as bifurcation parameter and $\tau_{1}=0$. Parameters values are same as Figure 4.

(a)

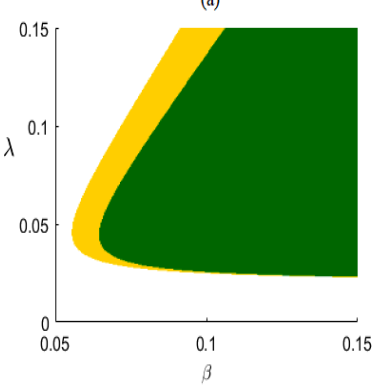

(b)

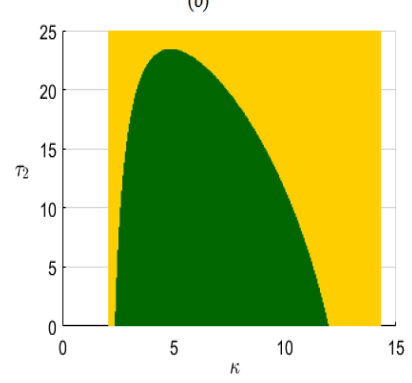

(c)

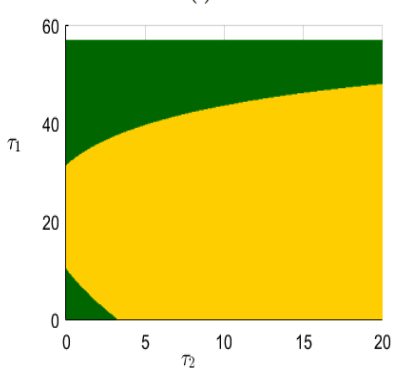

Fig. 6 (a) Region of stability of endemic equilibrium in $\beta-\lambda$ region, (b) Region of stability of endemic equilibrium $E^{*}$ in $\kappa-\tau_{2}$ parameter plane, (c) Region of stability of endemic equilibrium $E^{*}$ in $\tau_{1}-\tau_{2}$ parameter plane. Coexisting equilibrium $E^{*}$ is stable in green region and unstable in yellow region. In white region $E^{*}$ is not feasible.

The region of stability of endemic equilibrium $E^{*}$ is presented in Figure 6 . In Figure 6(a), stability of $E^{*}$ is shown in $\beta-\lambda$ parameter plane. We have observed the stabilizing role of $\beta$. It can be recognised that for lower values of both the parameters, $E^{*}$ is not feasible. For the higher value of $\lambda E^{*}$ is unstable but if the value of $\beta$ crosses a threshold value, $E^{*}$ becomes stable. Thus the critical value $\beta^{*}$ is dependent on the value of $\lambda$ and vice versa. Figure 6(b) shows that the effects of gestation delay $\tau_{2}$ is also dependent on other model parameters, for example, virus replication rate $\kappa$. It can be observed 
that for a larger value of $\kappa$, endemic equilibrium does not exist. In Figure 6(c), the region of stability in $\tau_{1}-\tau_{2}$ parameter plane is shown. This figure contains the combined effect of the two delays as discussed in Figure 4 and Figure 5.

\section{Discussion and Conclusion}

In this paper, a mathematical model using delay differential equations has been derived for the dynamics of integrated pest management (IPM) system using biopesticide and predatory insects as managing agents. In this system biopesticides (generally virus) attack the susceptible pest and make it infected after a latent period. We consider this period as latent delay $\tau_{1}$. We have also introduced predatory insects population in the model system. Infected pest are less harmful and also predators can consume the infected easily than susceptible pest.

We investigated the model system from the perspective of stability and persistence. We have explained the nonnegativity and boundedness of solutions of the proposed delayed model. We have obtained five equilibrium points. Using Routh-Hurtwiz criteria, we have derived their stability conditions. Numerically we have seen that the stability changes of endemic equilibrium point occurred through Hopf bifurcation. Finally, we have assumed that the infection of pests by biopesticide is a delayed process and modified the model accordingly. It is seen that the dual role of latent delay. It sometimes stabilizes the, but harmful for the system as the infected pest density decreases for increasing latent delay. In that case, we have to increase the rate of biopesticides release. Sometimes it is suggested to use chemical pesticides, i.e., pest culling [31,5]. Also, we have seen the destabilizing role of gestation delay. Consequently incorporation of additional food is recommended [26].

In conclusion, our work shows that spraying of pesticides along with the predatory insect is successful if this integrated approach is used correctly, i.e., considering the resistance in terms of latency of pest and gestation period (or maturation period of a predator). Hence time delay should be included in the modelling of a pest management system.

The present work can be extended in many ways. It can also be interesting how the enrichment of additional food for predator can effect the system. The pest maturation delay effect can also be studied.

\section{Conflict of Interest}

Authors declare that they have no conflict of interest.

\section{Data Availability}

The data used for this research is included within the article. 


\section{References}

1. P. DeBach, D. Rosen, Biological control by natural enemies, CUP Archive, (1991).

2. S. Ghosh, S. Bhattacharyya, D.K. Bhattacharya, The role of viral infection in pest control: a mathematical study. Bull. Math. Biol., 69 (2007), 2649-2691.

3. T. K. Kar A., Ghorai, S. Jana, Dynamics of pest and its predator model with disease in the pest and optimal use of pesticide, J. Theoret. Biol., 310 (2012) 187-198.

4. J. Chowdhury, F. A. Basir, J. Pal, P.K. Roy, Pest control for Jatropha curcas plant through viral disease: A mathematical approach, Nonlinear Studies., 23 (2016), 51732

5. Chowdhury, J., Al Basir, F., Takeuchi, Y., Ghosh, M. and Roy, P.K., A mathematical model for pest management in Jatropha curcas with integrated pesticides-An optimal control approach, Ecol. Complex., 37 ( 2019), 24-31.

6. Li, Jianjun, and Wenjie Gao, Analysis of a prey-predator model with disease in prey, Appl. Math. Comput.217 (2010): 4024-4035.

7. Tan, Y., Chen, L., Modelling approach for biological control of insect pest by releasing infected pest, Chaos, Solitons Fractals. 39, 2009, 304-315.

8. H. Zhang, L. Chen, J.J. Nieto, A delayed epidemic model with stage-structure and pulses for pest management strategy, Nonlinear Anal.: Real World Appl., 9, 1714-1726.

9. D. R. Dent, Integrated pest management and microbial insecticides in Microbial Insecticides: Novelty or Necessity?, In: Proceedings of the British Crop Protection Council Symposium, (1997) 127-138.

10. S. Jana, T.K. Kar, Modeling and analysis of a prey-predator system with disease in the prey, Chaos, Solitons 83 Fractals., 47 (2013) 42-53

11. S. Ghosh, S. Bhattacharyya, D.K. Bhattacharya, role of latency period in viral infection: A pest control model. Mathematical Biosciences, 210(2), pp.619-646.

12. V. Kumar, J. Dhar, H.S. Bhatti, H. Singh, Plant-pest-natural enemy dynamics with disease in pest and gestation delay for natural enemy, J. Math. Comput. Sci., 7 (2017), 948-965.

13. V. Kumar, J. Dhar, H.S. Bhatti, Stability Switching Dynamics of a Food Chain System Incorporating Gestation Delays, Diff. Eq. Dyn. Syst., (2019), 1-21.

14. G. M. Tatchell, Microbial insecticides and IPM: current and future opportunities for the use of biopesticides, BCPC Symposium Proceedings, 1997.

15. K. van Frankenhuyzen, R. C. Reardon, N. R. Dubois, Forest defoliators, Field Manual of Techniques in Invertebrate Pathology, Springer Netherlands, (2007) 481-504.

16. Bhattacharya D. K., Karan S., On bionomic model of integrated pest management of a single pest population, J. Differ. Equat. Dyn. Syst., 12(4), (2004), 301-330.

17. J. M. Franz, J. Huber, Field trials with insect pathogenic viruses in Europe, Entomophaga., 24(4), 1979 333-343.

18. Gopal, M., Gupta, A., Sathiamma, B., Nair, C.P.R., Control of the coconut pest Oryctes rhinoceros L. using the Oryctes virus. Insect Sci. Appl. 21(2), (2001) 93-101.

19. Naranjo, Steven E., Peter C. Ellsworth, and George B. Frisvold, Economic value of biological control in integrated pest management of managed plant systems, Annual review of entomology 60: 621-645, 2015.

20. Liang, J., Tanga, S., Cheke, R.A.: An integrated pest management model with delayed responses to pesticide applications and its threshold dynamics. Nonlinear Anal. Real World Appl. 13, 2352-2374 (2012)

21. Boethel, D.J., Eikenberry, R.D.: Interactions of Plant Resistance and Parasitoids and Predator of Insects.Wiley, Chichester (1986).

22. F. A. Basir, A. Banerjee, S. Ray, Role of farming awareness in crop pest management - A mathematical model, Journal of Theoretical Biology, 461 (2019) 59-67.

23. Bodnar M., The nonnegativity of solutions of delay differential equations, Applied Mathematics Letters, 13(6), (2000) 91-5.

24. X. Yang, L. Chen, J. Chen, Permanence and positive periodic solution for the single species nonautonomous delay diffusive model, Computers \& Mathematics with Applications, 32 (1996) 109-116.

25. Wang, X., Song, X., Mathematical models for the control of a pest population by infected pest, Comput. Math. Appl., 56 (2008) 266-278. 
26. Srinivasu, P.D.N., Prasad, B.S.R.V., Role of quantity of additional food to the predator as a control in predator-prey system with relevance to pest management and biological conservation, Bull. Math. Biol. 73, (2011) 2249-2276.

27. Luff, M. L., The potential of predators for pest control, Agriculture, ecosystems \& environment 10, no. 2 (1983): 159-181.

28. Jana, S. and Kar, T.K., 2013. A mathematical study of a prey-predator model in relevance to pest control. Nonlinear Dynamics, 74(3), pp.667-683.

29. Kumari, V. and Singh, N.P., 2009. Spodoptera litura nuclear polyhedrosis virus (NPV$\mathrm{S})$ as a component in Integrated Pest Management (IPM) of Spodoptera litura (Fab.) on cabbage. Journal of Biopesticides, 2(1), pp.84-86.

30. Singh, S.S. and Yadav, S.K., 2007. Comparative efficacy of insecticides, biopesticides and neem formulations against Helicoverpa armigera on chickpea. Annals of Plant Protection Sciences, 15(2), pp.299-302.

31. S.K. Sasmal, D. S. Mandal, J. Chattopadhyay, A predator-pest model with Allee effect and pest culling and additional food provision to the predator-application to pest control, J. Biological Systems 25 (2017), 295-326.

32. Misra, A.K., Tiwari, P.K., Chandra, P. Modeling the Control of Algal Bloom in a Lake by Applying Some External Efforts with Time Delay. Differ Equ Dyn Syst (2017). https://doi.org/10.1007/s12591-017-0383-5

\section{A Appendix}

The coefficients of (12) are given below:

$$
\begin{aligned}
l_{1}= & -F_{11}-F_{22}-F_{33}-F_{44} \\
l_{2}= & F_{11} F_{22}+F_{11} F_{33}+F_{22} F_{33}+F_{11} F_{44}+F_{22} F_{44}+F_{33} F_{44} \\
l_{3}= & -F_{11} F_{22} F_{33}-F_{11} F_{22} F_{44}-F_{11} F_{33} F_{44}-F_{22} F_{33} F_{44} \\
l_{4}= & F_{11} F_{22} F_{33} F_{44} \\
b_{1}= & -F_{12} G_{21}-F_{42} G_{24} \\
b_{2}= & F_{12} F_{33} G_{21}-F_{14} F_{42} G_{21}+F_{12} F_{44} G_{21}+F_{11} F_{42} G_{24}+F_{33} F_{42} G_{24} \\
b_{3}= & F_{14} F_{33} F_{42} G_{21}-F_{12} F_{33} F_{44} G_{21}-F_{11} F_{33} F_{42} G_{24}, \\
a_{1}= & -H_{33} \\
a_{2}= & -F_{13} H_{31}-F_{23} H_{32}+F_{11} H_{33}+F_{22} H_{33}+F_{44} H_{33} \\
a_{3}= & F_{13} F_{22} H_{31}-F_{12} F_{23} H_{31}+F_{13} F_{44} H_{31}+F_{11} F_{23} H_{32}+F_{23} F_{44} H_{32} \\
& -F_{11} F_{22} H_{33}-F_{11} F_{44} H_{33}-F_{22} F_{44} H_{33} \\
a_{4}= & -F_{14} F_{23} F_{42} H_{31}-F_{13} F_{22} F_{44} H_{31}+F_{11} F_{22} F_{44} H_{33}-F_{11} F_{23} F_{44} H_{32} \\
& +F_{12} F_{23} F_{44} H_{31}+F_{13} F_{42} G_{24} H_{31}, \\
q_{1}= & -F_{13} G_{21} H_{32}+F_{12} G_{21} H_{33}+F_{42} G_{24} H_{33} \\
q_{2}= & F_{13} F_{44} G_{21} H_{32}+F_{14} F_{42} G_{21} H_{33}-F_{12} F_{44} G_{21} H_{33}-F_{11} F_{42} G_{24} H_{33}
\end{aligned}
$$

\section{B Appendix}

For $\tau_{2}>0$ and $\tau_{1}=0$, the characteristic equation becomes

$$
\psi(\xi, \tau)=\xi^{4}+a_{1} \xi^{3}+a_{2} \xi^{2}+a_{3} \xi+a_{4}+e^{-\xi \tau}\left[b_{1} \xi^{3}+b_{2} \xi^{2}+b_{3} \xi+b_{4}\right] .
$$

For $\tau>0$, then (39) will have infinitely many roots. To determine the nature of the stability, the sign of the real parts of the roots of the characteristic equation (39) is required. A necessary condition for a stability changes of $E^{*}$ is that the characteristic equation (39) 
should have purely imaginary solutions. Let $i \zeta$ be a root of equation (39) and from which we get,

$$
\begin{aligned}
\zeta^{4}-a_{2} \zeta^{2}+a_{4} & =\left[\zeta^{2} b_{2}-b_{4}\right] \cos \zeta \tau-\left[\zeta b_{3}\right] \sin \zeta \tau \\
a_{1} \zeta^{3}-a_{3} \zeta & =\left[\zeta^{2} b_{2}-b_{4}\right] \sin \zeta \tau+\left[\zeta b_{3}\right] \cos \zeta \tau
\end{aligned}
$$

Squaring and adding above two equations,

$$
\zeta^{8}+\alpha_{1} \zeta^{6}+\alpha_{2} \zeta^{4}+\alpha_{3} \zeta^{2}+\alpha_{4}=0 .
$$

Simplifying and substituting $\zeta^{2}=l$ in equation (41) we get the following equation

$$
l^{4}+\alpha_{1} l^{3}+\alpha_{2} l^{2}+\alpha_{3} l+\alpha_{4}=0 .
$$

The roots of equation (42) have negative real parts if and only if its coefficients satisfy the Routh-Hurwitz criterion. In such case (39) does not have purely imaginary roots. Thus, we summarize the results in the following proposition.

Proposition 4 Suppose that the system without delay is stable. The endemic equilibrium $E^{*}$ is LAS for all $\tau_{2}>0$ if the following conditions are satisfied:

$$
\omega_{1}>0, \quad \omega_{4}>0, \quad \omega_{1} \omega_{2}-\omega_{3}>0, \quad\left(\omega_{1} \omega_{2}-\omega_{3}\right) \omega_{3}-\omega_{1}^{2} \omega_{4}>0 .
$$

If $\omega_{4}<0$ holds then equation (42) will admit at least one positive root. If $\theta_{0}^{2}$ is the minimum positive root of (42), then $\theta$ will be a purely imaginary root, $\pm i \theta_{0}$ corresponding to the delay $\tau_{2}$, then the endemic equilibrium $E^{*}$ remains stable for $\tau_{2}<\tau_{2}^{*}$.

We can evaluate the critical value of $\tau_{2}$ for which the endemic equilibrium $E^{*}$ remains stable. From equation (40)

$$
\tau_{2}^{*}=\frac{1}{\theta_{0}} \cos ^{-1}\left[\frac{b_{2}\left(-\theta_{0}^{4}+a_{2} \theta_{0}^{2}-a_{4}\right)+b_{1} a_{1} \theta_{0}^{3}}{b_{1}^{2}+b_{2}^{2}}\right]+\frac{2 \pi n}{\theta_{0}}, \quad n=0,1,2,3, \ldots
$$

From the above analysis, the following theorem follows.

Theorem 7 If $\omega_{4}<0$ is satisfied then the steady state $E^{*}$ is $L A S$ for $\tau_{2}<\tau_{2}^{*}$ and becomes unstable for $\tau_{2}>\tau_{2}^{*}$. Furthermore, the system will undergo a Hopf bifurcation at $E^{*}$ at $\tau_{2}=\tau_{2}^{*}$ provided

$$
4 \theta_{0}^{6}+A_{1} \theta_{0}^{4}+A_{2} \theta_{0}^{2}+A_{3} \neq 0
$$

where,

$$
A_{1}=3 a_{1}-6 a_{2}, \quad A_{2}=2 a_{2}+4 a_{4}-4 a_{1} a_{3}, \quad A_{3}=a_{3}^{2}-2 a_{2} a_{4}-b_{1}^{2} .
$$

Proof We need to prove the last conditions only. Now, differentiating (39) with respect to $\tau_{2}$ we get:

$$
\frac{d \tau_{2}}{d \xi}=\frac{4 \xi^{3}+3 a_{1} \xi^{2}+2 a_{2} \xi+a_{3}}{b_{1} \xi^{2}+b_{2}} e^{\xi \tau_{2}}+\frac{b_{1}}{b_{1} \xi^{2}+b_{2} \xi}-\frac{\tau_{2}}{\xi} .
$$

Now, using the relation (40) one can obtain:

$$
\begin{aligned}
\operatorname{sgn}\left[\frac{d(\operatorname{Re} \xi)}{d \tau_{2}}\right]_{\tau_{2}=\tau_{2}^{*}} & =\operatorname{sgn}\left[\operatorname{Re}\left(\frac{d \xi}{d \tau_{2}}\right)^{-1}\right]_{\xi=i \theta_{0}} \\
& =\operatorname{sgn}\left[\frac{4 \theta_{0}^{6}+A_{1} \theta_{0}^{4}+A_{2} \theta_{0}^{2}+A_{3}}{b_{1} \theta_{0}^{2}+b_{2}^{2}}\right]
\end{aligned}
$$

and the latter is positive if $4 \theta_{0}^{6}+A_{1} \theta_{0}^{4}+A_{2} \theta_{0}^{2}+A_{3} \neq 0$ i.e. the transversality condition holds and the system undergoes Hopf bifurcation at $\tau_{2}=\tau_{2}^{*}$. 